\title{
A review of representation models of tolerance information
}

\author{
Yuchu Qin ${ }^{1} \cdot$ Qunfen $\mathrm{Qi}^{2} \cdot$ Wenlong Lu ${ }^{1} \cdot$ Xiaojun $\mathrm{Liu}^{1} \cdot$ Paul J. Scott ${ }^{2} \cdot$ Xiangqian Jiang $^{2}$
}

Received: 5 July 2017 / Accepted: 9 November 2017 / Published online: 21 November 2017

(C) The Author(s) 2017. This article is an open access publication

\begin{abstract}
A good representation model of tolerance information is indispensable for achieving computer-aided tolerancing. Currently, the representation model used in industry is the EXPRESS model, while at the same time, a number of other kinds of representation models have been presented within the academia. The coexistence of different kinds of representation models generates a series of questions and discussions: can the EXPRESS model completely implement the representation of tolerance information semantics in an explicit, computer-readable, and computer-interpretable way? What challenges have been addressed to date by the presented representation models? What are the advantages and disadvantages of each representation model? What capabilities should an ideal representation model have? What are the potential research directions in tolerance information representation in the future? To approach these questions, a review of representation models of tolerance information is presented in this paper. An in-depth analysis of existing representation models is firstly provided. Then, the review makes a detailed comparison among them based on this analysis. Finally, some future research directions in tolerance information representation are suggested.
\end{abstract}

Keywords Tolerance information $\cdot$ Representation model $\cdot$ EXPRESS model $\cdot$ Semantics $\cdot$ Design requirement

\section{Introduction}

Computer-aided tolerancing (CAT) is the use of computer systems to aid in the design and optimization of the dimensions and tolerances of product components [1]. To achieve CAT, the tolerance information presented in technical handbooks and tolerancing standards should be firstly interpreted in an unambiguous and rigorous way. Then, the interpreted tolerance information requires being represented in an explicit, computer-readable, and computer-interpretable way. After implementing the represented tolerance information in computer systems, CAT can be carried out. In such a process (whose schematic representation is shown in Fig. 1), two kinds of tolerance information models need to be established. They are interpretation and representation models.

Interpretation model aims at interpreting the semantics (i.e., meaning) of the presented tolerance information in an

Qunfen Qi

q.qi@hud.ac.uk

1 The State Key Laboratory of Digital Manufacturing Equipment and Technology, School of Mechanical Science and Engineering,

Huazhong University of Science and Technology, Wuhan 430074, People's Republic of China

2 EPSRC Future Advanced Metrology Hub, University of Huddersfield, Huddersfield HD1 3DH, UK unambiguous and rigorous way. It is also called mathematical model since it is usually formulated by mathematical languages and described in the form of mathematical expressions. During the past few decades, a variety of interpretation models, such as offsetting model [2-4], parametric model $[5-7]$, vector equation model $[8,9]$, variational surface model [10], kinematic model [11-13], generalized interval model [14], degree of freedom (DOF) model [15-17], and tolerance-map model [18-20], have been proposed. Benefited from the gaining popularity, in the same period, researchers have also carried out extensive reviews upon this subject, such as the reviews of Liu et al. [21], Hong and Chang [22], Marziale and Polini [23-25], Ameta et al. [26], Polini [27], and Chen et al. [28].

Representation model is used to represent the semantics of the interpreted tolerance information and is usually established on the basis of an interpretation model. Currently, the representation model used in industry is the EXPRESS model [29-31], while at the same time, other representation models, such as surface graph model [32-34], representational primitive model $[35,36]$, technologically and topologically related surface (TTRS) model [37], unified modeling language (UML) model [38], extensive markup language (XML) model [39], category theory model [40-43], GeoSpelling model [44], relationship model $[45,46]$, and ontology-based model [47-50], have been presented within the academia. The coexistence of different kinds of representation models triggers a 
Fig. 1 General process of achieving CAT

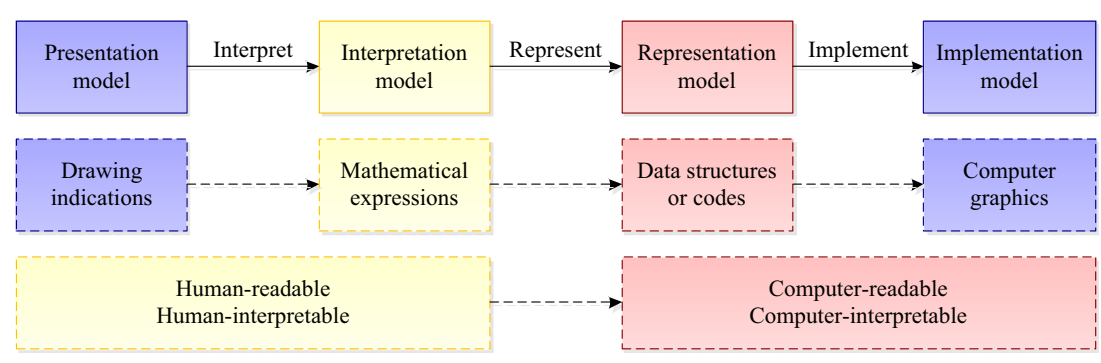

series of questions and discussions within the academia and the industry. Yet, Q1: Can the EXPRESS model completely implement the representation of tolerance information semantics in an explicit, computer-readable, and computerinterpretable way? Q2: What challenges have been addressed to date by the presented representation models? Q3: What are the advantages and disadvantages of each representation model? Q4: What capabilities should an ideal representation model have? Q5: What are the potential research directions in tolerance information representation in the future?

This paper attempts to approach these questions via presenting a review of representation models of tolerance information. Although Roy et al. [51], Juster [33], Yu et al. [52], Liu et al. [21], and Hong and Chang [22] have, respectively, presented related reviews, the review in the present paper is still of necessity because it covers more representation models than these five related reviews. To be more specific, the five related reviews were made at least 15 years ago and do not include the new representation models that have emerged during this period. The reviews in reference [33, 51, 52] only refer to the surface graph model, while the reviews in reference $[21,22]$ just involve the surface graph, representational primitive, and TTRS models. Compared to the five related reviews, the review in the paper includes not only the surface graph, representational primitive, and TTRS models, but also the EXPRESS, UML, XML, category theory, GeoSpelling, relationship, and ontology-based models.
The remainder of the paper is organized as follows. Section 2 provides an introduction and analysis of existing representation models of tolerance information. A comparison between these representation models is presented in Sect. 3. Some future research directions in tolerance information representation are discussed in Sect. 4. Section 5 ends the paper with a conclusion.

\section{Tolerance information representation models}

A review is started from the first three research questions Q1, Q2, and Q3. This section attempts to approach these questions through an introduction and analysis of the tolerance information representation models listed in Table 1. According to Table 1, the section is divided into ten subsections: EXPRESS model, surface graph model, representational primitive model, TTRS model, UML model, XML model, category theory model, GeoSpelling model, relationship model, and ontology-based model.

\subsection{EXPRESS model}

The EXPRESS model was established by the International Standardization Organization (ISO) to implement the archiving of tolerance information in computer systems and the exchange of tolerance information among heterogeneous
Table 1 Ten different kinds of tolerance information representation models in the literature

\begin{tabular}{lll}
\hline Representation model & Representative scholars and corresponding references & Notation \\
\hline EXPRESS model & ISO 10303-203 [29]; ISO 10303-214 [30]; ISO 10303-242 [31] & RM1 \\
Surface graph model & Requicha and Voelcker [32]; Juster [33]; Roy and Liu [34] & RM2 \\
$\begin{array}{l}\text { Representational primitive } \\
\text { model }\end{array}$ & Guilford and Turner [35]; Wilhelm and Lu [36] & RM3 \\
TTRS model & Desrochers and Clement [37] & RM4 \\
UML model & Rachuri et al. [38] & RM5 \\
XML model & Zhao et al. [39] & RM6 \\
Category theory model & Lu et al. [40], Xu et al. [41], Qi et al. [42, 43] & RM7 \\
GeoSpelling model & Dantan et al. [44] & RM8 \\
Relationship model & Zhang et al. [45]; Qin et al. [46] & RM9 \\
Ontology-based model & Fiorentini et al. [47]; Lu et al. [48]; Qin et al. [49, 50] & RM10 \\
\hline
\end{tabular}


computer systems [53]. The established process is roughly as follows:

In 1994, an overview of the STEP (standard for the exchange of product model data) standard system [54] was published by ISO. In this overview, the STEP standard system was classified into nine groups of description methods, implementation methods, conformance testing methodology and framework, abstract test suites, integrated generic resources, integrated application resources, application interpreted constructs, application protocols (APs), and application modules, where ISO 10303-203 in the group of APs (which was also published in 1994 and generally known as AP 203 1994) was soon implemented in commercial computer-aided design (CAD) systems. AP 203 1994, which specified the integrated resources required in the application systems of configuration-controlled 3D designs of mechanical parts and assemblies, did not involve the representation of tolerance information. To specify the integrated resources required in the application systems supporting the development process of the mechanical aspects of automotive vehicles, ISO 10303-214 (generally known as AP 214 2001) was published in 2001. Like AP 203 1994, AP 2142001 also did not include the representation of tolerance information. This AP was soon revised, and its second edition (i.e., AP 214 2003) was published in 2003. The representation of dimensional information and geometrical tolerance information was available in this edition. In 2010, AP 214 was revised again and its third edition (i.e., AP 214 2010) was published. A year later, the second edition of AP 203 (i.e., AP 203 2011) was published. Compared to its first edition, AP 2032011 added the representations of a number of types of product information, where the information of geometric and dimensional tolerances is one of them. To represent and exchange a wider range of product information throughout the whole product life cycle, ISO completed a major development on a new AP entitled "Managed ModelBased 3D Engineering" by merging and extending AP 2032011 and AP 2142010 in 2014. This new AP was published as AP 2422014.

At present, the EXPRESS models that have been widely used in industry are the tolerance information representation models in AP 2032011 [29], AP 2142010 [30], and AP 242 2014 [31]. These models firstly define the EXPRESS schema [55] of each type of tolerance in an object-oriented manner. They then use concrete tolerance information to instantiate the predefined EXPRESS schema and thus a STEP file is generated. This file is encoded in a form of clear text [56], which can be read by any STEP file analyzers and almost all commercial CAD systems.

The EXPRESS model can provide a syntactically consistent representation model of tolerance information (i.e., the model is computer-readable), which satisfies the requirements of the representation of tolerance information syntax in computer systems and the exchange of the syntax among heterogeneous computer systems. However, the model cannot meet the demand of the representation and exchange of tolerance information semantics (i.e., the model is not really computerinterpretable), because EXPRESS is unable to explicitly represent the semantics [57] and the model will not contain any representation of tolerance information semantics [58].

\subsection{Surface graph model}

The surface graph model takes the geometric structure of component as the fundamental structure of surface graph, takes the geometric features, surfaces, edges, and vertices of component as the nodes of surface graph, and takes the datum and tolerances of component as the attributes of the nodes of surface graph. Representative examples of this kind of model are the constructive solid geometry (CSG) model presented by Requicha and Voelcker [32], the boundary-representation (B-Rep) model presented by Juster [33], and the CSG/B-Rep hybrid model presented by Roy and Liu [34].

The surface graph model can provide a data structure to represent tolerance information. But it has not yet addressed the issue of representing tolerance information semantics.

\subsection{Representational primitive model}

The representational primitive model was firstly presented by Guilford and Turner [35]. It uses classes to describe the representational primitives of tolerance information and uses attributes of classes to describe tolerance information. By using functions of classes to instantiate attributes of classes, the representation of tolerance information can be implemented. On the basis of Guilford and Turner's model, Wilhelm and Lu [36] proposed the conditional tolerances based on representational primitives in concurrent engineering and adopted these representational primitives to represent tolerance information.

The representational primitive model is capable of representing most types of tolerances in tolerancing standards via certain representational primitives and their combinations. However, it only adds the tolerance information to the corresponding geometric features and does not really represent the semantics of tolerance information.

\subsection{TTRS model}

The TTRS model was presented by Desrochers and Clement [37]. It firstly organizes the functional surfaces of component as TTRS binary trees, then establishes the minimum geometric datum element (MGDE) of each TTRS, and finally represents tolerance information via MGDEs and the relationships between them. The biggest characteristic of the model is 
the reorganization of the geometric information provided by the computer systems, which can lay a foundation for implementing automatic tolerancing in computer systems. However, the model mainly considers topologically related surfaces and does not really consider technologically related surfaces [21].

Aiming at the issues that the TTRS model does not consider technologically related surfaces, Liu et al. [59] extended the TTRS model and constructed a feature-based TTRS (FTTRS) model. This model firstly establishes the TTRS of each geometric feature, the minimum feature datum element (MFDE) of each FTTRS, and the constraint primitives of each MFDE. It then represents tolerance information via MFDEs and their constraint primitives. The FTTRS model implements the representation of tolerance information according to semantics and simultaneously considers topologically and technologically related surfaces. However, the number of feature types that can be processed by the model is somewhat limited [21].

In addition to the extension of the TTRS model, the application of the model is also gaining importance and popularity within the industry and the academia. As described in an overview of current CAT systems presented by Prisco and Giorleo [60], the model was used to represent the tolerance information in the commercial CAT system CATIA.3D FDT ${ }^{\text {TM }}$. Based on the TTRS model, Clement et al. [61] presented a method for computer-aided dimensioning and tolerancing; Desrochers and Maranzana [62] designed rules for selecting MGDEs; Salomons et al. [63] developed a CAT system; Toulorge et al. [64] presented a digital functional assistance process for tolerancing; Jaballi et al. [65] proposed a rational method for 3D manufacturing tolerancing synthesis; and Zhang et al. [66] designed rules for selecting datum and validating datum reference frame.

\subsection{UML model}

The UML model was established by Rachuri et al. [38], which can be seen as an extension of the core product model [67] (it was obtained via adding the UML representations of tolerance information, tolerance propagation information, kinematics information, and engineering analysis information to the core product model). The model firstly leverages UML to describe dimension and geometric tolerances. Then, through creating UML instances for concrete tolerance information, the representation can be implemented.

The UML model can provide a way for tolerance information representation at the system level. It is open so as to enable plug-and-play with various computer systems. However, the model has not yet provided the representation of variational geometric constraint information and tolerance zone information. Besides, it is not really computer-interpretable, because UML is not based on formal semantics.

\subsection{XML model}

The XML model was presented by Zhao et al. [39]. It firstly abstracts the explanations and illustrations of each type of tolerances in the American Society of Mechanical Engineers (ASME) tolerancing standard Y14.5M-1994 [68], then transforms the abstracted explanations and illustrations into XML schemas, and finally uses the tolerance information in CAD systems to instantiate the XML schemas. By this way, the computer representation of the tolerance information for integrated measurement processes is implemented.

The XML model can act as an adapter for the unambiguous communication of tolerance information via the Internet among different application domains. Further, the XML itself has been extended to represent semantics explicitly by a number of researchers. As an example, Liu et al. [69] proposed an XML semantics definition language to express XML schemas' intended meaning and proposed a model-theoretic semantics for XML. However, the XML model has not yet included the representation of the semantics of tolerance information, because the abstracted explanations and illustrations in ASME Y14.5M-1994 just provide a definition of different types of tolerances and do not involve such semantics.

\subsection{Category theory model}

The category theory model was firstly presented by Wang et al. [70] to represent the knowledge of profile surface texture. It has later been applied by Lu et al. [40] to systematically describe the information of geometrical product specifications (GPS) via certain categorical constructors in category theory, which include category, subcategory, object, morphism, instance, pull back, product, functor, and natural transformation. The model provides an abstract representation of the tolerance specifications described in natural language in technical handbooks and tolerancing standards.

On the basis of Wang et al.'s model on profile surface texture, Xu et al. [41] and Qi et al. [42, 43] have further developed and implemented the category theory model on surface texture; as a result, they successively developed knowledge-based systems for the manipulation of complex GPS information. The systems enable mechanical designers to query specific rules to design tolerance specifications. The ambiguous issue caused by describing tolerance specifications in natural language can be well addressed. The systems also provide a structural mapping from the design model to the inspection model, such that a measurement plan can be generated from its specification requirement.

The category theory model, though, has great potential to represent the semantics of tolerance information; at current stage, it has not yet been fully developed to include the representation of tolerance information semantics. The reason is as 
follows: category theory is an abstract form of mathematical language focusing on how things behave. It can provide an effective and natural formalism to describe things and their relationships and a powerful way to model complex systems with heterogeneous structures. But currently, category theorybased modeling still stays in an enabling fashion. There is not a universal semantic representation language for it. To this end, category ontology language, a new semantic representation language whose mathematical basis is category theory, is currently under development by Qi et al. at the University of Huddersfield. After developing this language, the representation of tolerance information semantics can be implemented.

\subsection{GeoSpelling model}

To define a coherent expression of GPS information during the tolerancing process along product life cycle, Dantan et al. [44] presented the GeoSpelling model. They firstly analyzed the requirements for preliminary design, then defined the concepts geometric feature, operation, characteristic, and condition for the model, and finally used the concepts to describe concrete tolerance information. The GeoSpelling model allows the communication of tolerance information in design, manufacturing, or inspection. It can provide the possibility to clearly distinguish the geometric elements manipulated during tolerance specification, inspection, and analysis.

To formally represent the GeoSpelling model, Ballu et al. [71] developed a computer-readable formal language. The syntax of this language is based on the function, condition, and loop in programming language, where function stands for manipulation and declaration of loop, condition stands for selecting specific geometric features from a set of geometric features, and loop stands for managing a set of geometric features. Through such syntax, the language is expected to be applied to represent the tolerance information in simulation metrology, assembly sequence, or manufacturing sequence. But there is yet no evidence to illustrate such application.

\subsection{Relationship model}

The relationship model uses certain relationship modeling methods to express the binary relationships between certain geometric elements and tolerances. The tolerance information can be represented via such expression. Representative examples of this kind of model are the polychromatic set model [45] and the adjacency matrix model [46].

The polychromatic set model was presented by Zhang et al. [45]. This model is based on the variational geometric constraint network theory [72] and is hierarchically organized. It consists of five layers of assembly layer, part layer, assembly feature surface layer, assembly tolerance specification layer, and assembly tolerance zone layer. The relationships in each layer are represented by one or more polychromatic sets.
Based on the reasoning capability of polychromatic sets, Zhang et al. [73] designed a new reasoning algorithm for assembly tolerance specifications and corresponding tolerance zone types. Similar to the polychromatic set model, the adjacency matrix model, which was presented by Qin et al. [46], is also hierarchically organized. It consists of four layers of part layer, assembly feature surface layer, spatial relation layer, and assembly tolerance type layer. The relationships in each layer are represented by one or more adjacency matrices. On the basis of such representations, an algorithm for generating assembly tolerance types was designed.

The biggest advantage of the relationship model is that it is simple, intuitive, and easy to implement. But the model just represents some corresponding relationships in tolerance information and does not represent the semantics.

\subsection{Ontology-based model}

To make the UML model [38] computer-interpretable, Fiorentini et al. [47] presented a web ontology language (OWL) [74] ontology version of the model. They also introduced the semantic web rule language (SWRL) [75] to the ontology and implemented the automatic reasoning on the ontology supported by OWL and SWRL. Due to the rigorous logic-based semantics of OWL, the OWL ontology version of the UML model was expected to implement the exchange of tolerance information semantics among different phases of product life cycle. But it has not yet provided the representation of variational geometric constraint information and tolerance zone information, because the UML model does not include such representation.

Inspired by Fiorentini et al. work, Lu et al. [48] provided the OWL ontology representation of variational geometric constraint information, and Qin et al. [49, 50] provided the OWL ontology representation of tolerance zone information and composite tolerance information. In addition to using OWL ontology to achieve tolerance information representation, Zhong et al. [76, 77] used OWL ontology to study the automatic generation of assembly tolerance types in CAD systems; Ahmed and Han [78] leveraged OWL to implement the exchange of the tolerance information indicated in CAD systems among heterogeneous CAD systems; and Qin et al. [79] designed an OWL ontology supported case-based reasoning approach to assist tolerance specification.

OWL ontology has advantages in the aspects of conformance checking, computer-readable and computer-interpretable, representation and exchange capabilities of semantics, and logic-based inference capability [80]. Thus, it comes as no surprise that the application of OWL ontologies in the representation of product information (including tolerance information) is gaining importance and popularity within the academia [81-85]. However, the OWL ontology-based model is probably not an efficient representation model for tolerance 
information as a whole, because OWL ontology has drawbacks in the aspects of degree of automation, time complexity, additional work, and negation in the model [80].

\section{Comparison}

A comparison is started from the penultimate research question Q4. This section attempts to approach this question via a comparison among the ten different kinds of representation models in Table 1. The details of this comparison are shown in Table 2. A quick study of Table 2 reveals that the comparison can be made from the following 12 aspects:

- Is the representation model directly computer-readable? The EXPRESS, XML, and ontology-based models are directly computer-readable, since their physical manifestations are, respectively, a STEP file, an XML file, and an OWL file, and each file consists of a set of computerreadable codes. Correspondingly, the surface graph, representational primitive, TTRS, UML, category theory, GeoSpelling, and relationship models are not directly computer-readable, because their physical manifestations are abstract data structures, whose computer readability is achieved by the aid of certain computer programming languages.

- Is the representation model directly computerinterpretable? If a model is directly computer-interpretable, this model must be directly computer-readable. Hence, the surface graph, representational primitive, TTRS, UML, category theory, GeoSpelling, and relationship models are not directly computer-interpretable (since they are not directly computer-readable). As for the EXPRESS, XML, and ontology-based models, only the ontology-based model is directly computer-interpretable, because OWL has formal semantics as its foundation (the formal semantics of OWL is based on the formal semantics of description logics [86], a family of knowledge representation and reasoning languages) and both EXPRESS and XML are not based on formal semantics.

- Has the representation model unambiguously represented information syntax? It is no doubt that each of the ten different kinds of representation models has unambiguously represented the syntax of tolerance information, since unambiguous syntax representation capability is the most fundamental capability of a representation model.

- Has the representation model explicitly represented information semantics? Explicitly representing the semantics of information requires capturing such semantics and using specific representation tools to represent the captured semantics. The ontology-based model has captured the detailed semantics of tolerance information and used OWL [74], an ontology representation language based on the formal semantics of description logics [86] and developed for the Semantic Web [87], to represent the captured semantics. As an example, Fig. 2 shows a composite positional tolerance specified on a rectangular pattern of holes [50]. The detailed semantics of this composite positional tolerance can be informally described as follows: (1) In the feature control frame of the composite positional tolerance, one positional tolerance symbol is assigned to two horizontal segments. As depicted in Fig. 3, the upper
Table 2 A comparison among the ten different kinds of tolerance information representation models in the literature

\begin{tabular}{lllllllllll}
\hline Comparison aspect & RM1 & RM2 & RM3 & RM4 & RM5 & RM6 & RM7 & RM8 & RM9 & RM10 \\
\hline Aspect 1 & YES & NO & NO & NO & NO & YES & NO & NO & NO & YES \\
Aspect 2 & NO & NO & NO & NO & NO & NO & NO & NO & NO & YES \\
Aspect 3 & YES & YES & YES & YES & YES & YES & YES & YES & YES & YES \\
Aspect 4 & DK & DK & DK & DK & DK & DK & DK & DK & DK & YES \\
Aspect 5 & YES & YES & YES & YES & YES & YES & YES & YES & YES & YES \\
Aspect 6 & NO & NO & NO & NO & NO & NO & NO & NO & NO & NO \\
Aspect 7 & YES & DK & DK & YES & DK & DK & DK & DK & DK & DK \\
Aspect 8 & YES & NO & NO & NO & YES & YES & NO & NO & NO & YES \\
Aspect 9 & YES & NO & NO & NO & NO & YES & NO & NO & NO & YES \\
Aspect 10 & YES & DK & DK & DK & YES & YES & DK & DK & DK & YES \\
Aspect 11 & NO & NO & NO & NO & NO & NO & NO & NO & NO & YES \\
Aspect 12 & NO & NO & NO & NO & NO & NO & NO & NO & NO & NO \\
\hline
\end{tabular}

Notes: Aspect 1: is it directly computer-readable? Aspect 2: is it directly computer-interpretable? Aspect 3: has it unambiguously represented information syntax? Aspect 4: has it explicitly represented information semantics? Aspect 5: has it included a systematic representation of product geometry? Aspect 6: has it included a systematic representation of design requirements? Aspect 7: has it been applied in industry? Aspect 8: does it adopt a universal tool to represent information? Aspect 9: does it provide an interface for information exchange? Aspect 10: is it easy to be reused and extended? Aspect 11: does it have intrinsic conformance checking, query, and reasoning capabilities? Aspect 12: does it have self-learning and automatic update capabilities? 
Fig. 2 A composite positional tolerance specified on a rectangular pattern of holes [50]

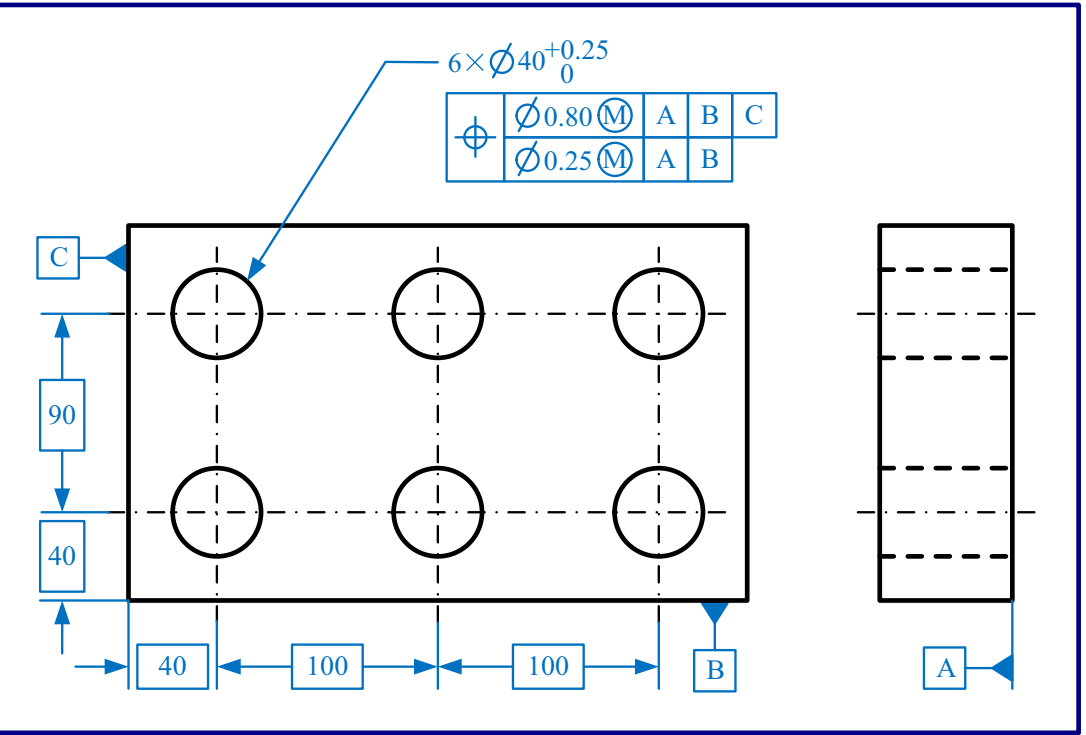

segment establishes a pattern-locating tolerance zone framework (PLTZF) $C_{1,1} C_{1,2} \ldots C_{1,6}$ that governs the relationship between the datum planes $\mathrm{A}, \mathrm{B}$, and $\mathrm{C}$ and the pattern, and the lower segment establishes a feature-relating tolerance zone framework (FRTZF) $C_{2,1} C_{2,2} \ldots C_{2,6}$ that is a refinement of the PLTZF and governs the relationship between features. (2) Letter $A$ in the upper segment orients the axes of the six $\phi 0.80$ cylinder tolerance zones $C_{1,1}, C_{1,2}, \ldots, C_{1,6}$ perpendicular to datum plane A. Letters $B$ in the upper segment locates the axes of $C_{1,1}, C_{1,2}$, and $C_{1,3}$ with $130 \mathrm{~mm}$ to datum plane B and locates the axis of $C_{1,4}, C_{1,5}$, and $C_{1,6}$ with $40 \mathrm{~mm}$ to datum plane $B$. Letter $C$ in the upper segment locates the axes of $C_{1,1}$ and $C_{1,4}$ with $40 \mathrm{~mm}$ to datum plane C, locates the axes of $C_{1,2}$ and $C_{1,5}$ with $140 \mathrm{~mm}$ to datum plane $\mathrm{C}$, and locates the axes of $C_{1,3}$ and $C_{1,6}$ with $240 \mathrm{~mm}$ to datum plane C. (3) The FRTZF controls the cylinder tolerance zones $C_{2,1}, C_{2,2}, \ldots, C_{2,6}$ within the tighter tolerance value $\phi 0.25$ and controls the location of the axes of $C_{2,1}, C_{2,2}, \ldots, C_{2,6}$ with 90 or $100 \mathrm{~mm}$ to each other. Letter $A$ in the lower segment orients the axes of $C_{2,1}, C_{2,2}, \ldots, C_{2,6}$ perpendicular to datum plane A. Letter $\mathrm{B}$ in the lower segment orients the axes of $C_{2,1}, C_{2,2}$, $\ldots, C_{2,6}$ parallel to datum plane $\mathrm{B}$. The FRTZF is free to translate within the boundaries governed by the PLTZF. (4) As also depicted in Fig. 3, a maximum material condition (MMC) is applied to the two positional tolerances $\phi 0.80$ and $\phi 0.25$ of each hole. This means that the dimensional and positional tolerances of each hole satisfy the MMC. For example, the meaning of the MMC applied
Fig. 3 The semantics of the composite positional tolerance [50]

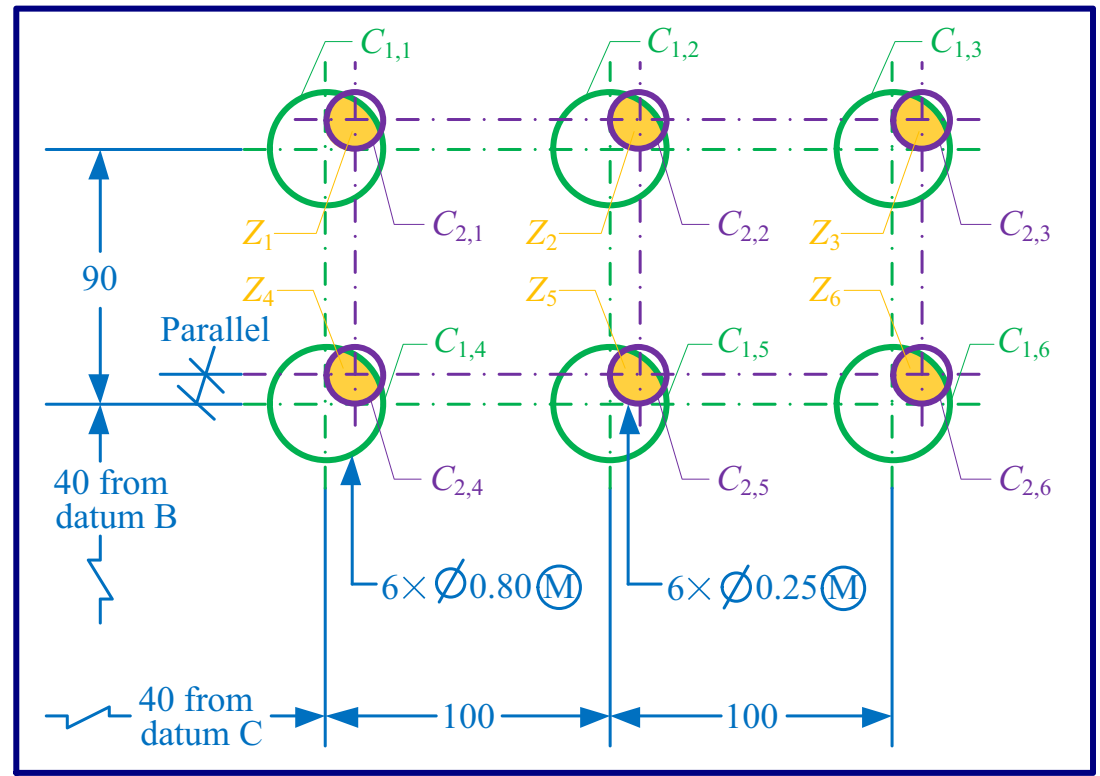


to the positional tolerance whose tolerance zone is $C_{1,1}$, as depicted in Fig. 4, can be described as follows: (1) When $C_{1,1}$ is at MMC, the external function size of the actual hole must be greater than or equal to the maximum material virtual size $\phi 39.2$. When everywhere of the local actual size of the hole is the maximum material size $\phi 40$, the maximum allowable value of the positional error of the axis is $\phi 0.80$. (2) When $C_{1,1}$ is not at MMC, the positional error of the axis can be greater than $\phi 0.80$. For instance, when everywhere of the local actual size of the hole is the minimum material size $\phi 40.25$, the maximum allowable value of the positional error of the axis is $\phi 1.05$. (3) The local actual size of the hole must lie between $\phi 40$ and $\phi 40.25$. The semantics of the MMC applied to the positional tolerances whose tolerance zones are each of $C_{1,2}, C_{1,3}, \ldots, C_{1,6}, C_{2,1}, C_{2,2}, \ldots, C_{2,6}$ can be described in a similar way. (5) The resultant tolerance zone of the composite positional tolerance is the intersection of its PLTZF and FRTZF (e.g., the intersection shown in Fig. 3). To satisfy the requirement of the composite positional tolerance, the variational geometry of each hole must lie in the resultant tolerance zone. The semantics described above have been represented using OWL in the ontology-based model. For the details of such representation, please refer to reference [50]. Correspondingly, there is yet no evidence that the EXPRESS, surface graph, representational primitive, TTRS, UML, XML, category theory, GeoSpelling, and relationship models have captured the detailed semantics of tolerance information. Thus, it is not clear whether these models have explicitly represented the semantics of tolerance information.

- Has the representation model included a systematic representation of product geometry? Tolerance information representation needs to synthetically consider the information of product geometry, design requirements (i.e., functional and assembly requirements), process (e.g., assembly sequence, fixture), and material, where the information of product geometry is the most fundamental information and the information of design requirements is the most important information [88]. It is no doubt that each of the ten different kinds of representation models has, respectively, provided a systematic representation of product geometry.

- Has the representation model included a systematic representation of design requirements? Considering the representation of design requirements is very important for tolerance information representation, because both tolerancing and assembly serve the purpose of fulfilling the function requirements of products. Even though some single tolerancing cases (e.g., the tolerancing cases presented by Ballu and Mathieu [89], Wang et al. [90], Anselmetti [91], Armillotta [92], and Cao et al. [93]) have involved it, systematically considering design requirements in tolerancing remains a challenging objective. There is no evidence to show that any representation models of tolerance information have included a systematic representation of design requirements.

- Has the representation model been applied in industry? Among the ten different kinds of representation models, the EXPRESS model has been widely used to implement the representation, archiving, and exchange of tolerance information in industry [53]. Almost all of the current commercial CAD systems support the import and export of STEP file, the physical manifestation of the EXPRESS model. A special forum called CAx Implementor Forum [94], whose participants are a group of CAD system developers and testers, was established to work on the industry application of the EXPRESS model. Except the
Fig. 4 The semantics of the MMC applied on the positional tolerance whose tolerance zone is $C_{1, i}(i=1,2, \ldots, 6)[50]$
Tolerance zone when $C_{1, i}$ is at $\mathrm{MMC}$

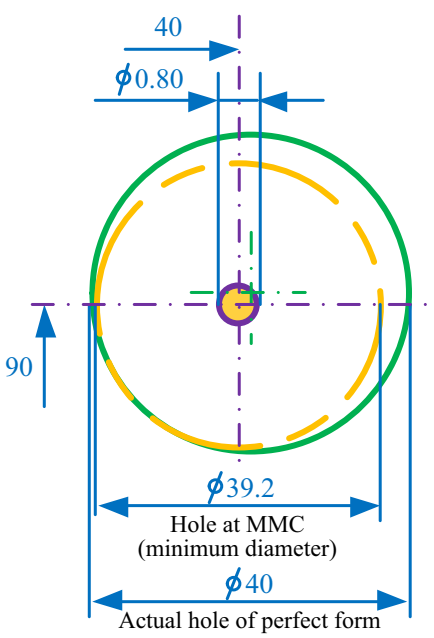

Tolerance zone when $C_{1, i}$ is not at MMC

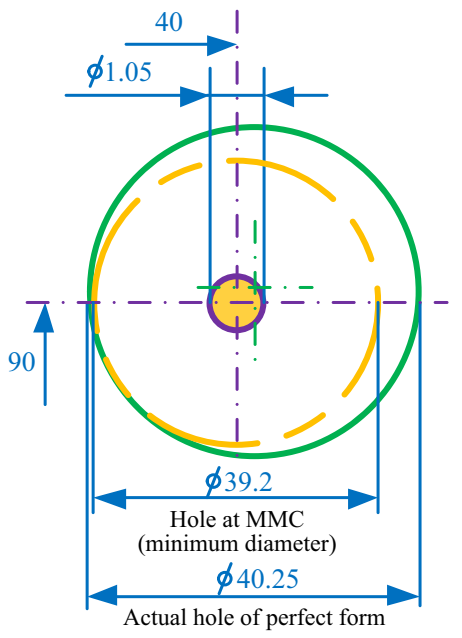




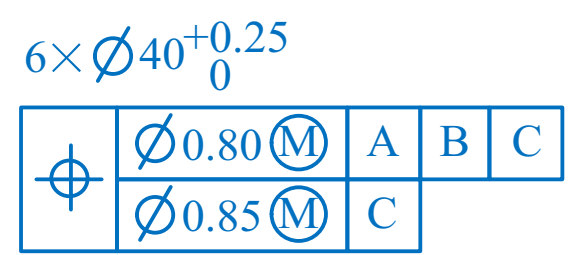

Fig. 5 An inconsistent drawing indication of a composite positional tolerance [50]

EXPRESS model, the TTRS model has also been applied in industry. It was used to represent the tolerance information in the commercial CAT system CATIA.3D FDT ${ }^{\text {TM }}$ [60]. As for the remaining eight kinds of representation models, there is yet no evidence that they have been used in industry.

- Does the representation model adopt a universal representation tool to represent information? The representation tools of the ten different kinds of representation models are, respectively, EXPRESS, graph, representational primitive, binary tree, UML, XML, category theory, GeoSpelling formal language, polychromatic set (adjacency matrix), and OWL. Among these representation tools, EXPRESS [55] is a standard data modeling language that has been widely used to represent product data in computer systems; UML [95] is a standard modeling language that has been widely used to visualize the design of a system in the domain of software engineering; XML [96] is a standard markup language that has been widely used to encode documents in a computer-readable format across the Internet; and OWL [74] is a standard ontology representation language that has been widely used to encode ontologies in a computer-readable and computerinterpretable format in various domains. Relatively speaking, these four representation tools are more universal than the remaining six representation tools.

- Does the representation model provide an interface for information exchange? The tolerance information in a representation model can be directly exchanged among heterogeneous computer systems if the physical manifestation of this representation model is directly computerreadable. Since the physical manifestations of the EXPRESS, XML, and ontology-based models are directly computer-readable, these three representation models provide interfaces for the exchange of tolerance information. Actually, the three representation models have been used in tolerance information exchange by Venkiteswaran et al. [97], Baysal et al. [98], and Ahmed and Han [78], respectively.

- Is the representation model easy to be reused and extended? Generally, a representation model is easy to be reused and extended by others if it adopts a universal representation tool to represent information, because a universal representation tool is easier to obtain and its learning materials are more complete. As the EXPRESS, UML, XML, and ontology-based models adopt relatively universal representation tools, these representation models are easier to be reused and extended by others.

- Does the representation model have intrinsic conformance checking, query, and reasoning capabilities? Whether a representation model has intrinsic conformance checking, query, and reasoning capabilities depends on the intrinsic conformance checking, query, and reasoning capabilities of its representation tool. Among the representation tools of the ten different kinds of representation models, only OWL has intrinsic conformance checking, query, and reasoning capabilities, since it is based on the formal semantics of description logics [86], a family of knowledge representation and reasoning languages. The intrinsic conformance checking, query, and reasoning on the ontology-based model can be implemented using description logic consistency decision algorithm, description logic query, and description logic reasoning, respectively. For example, Fig. 5 shows an inconsistent drawing indication of composite positional tolerance [50]. The inconsistent places of this drawing indication are in the indications of tolerance value and datum feature in the

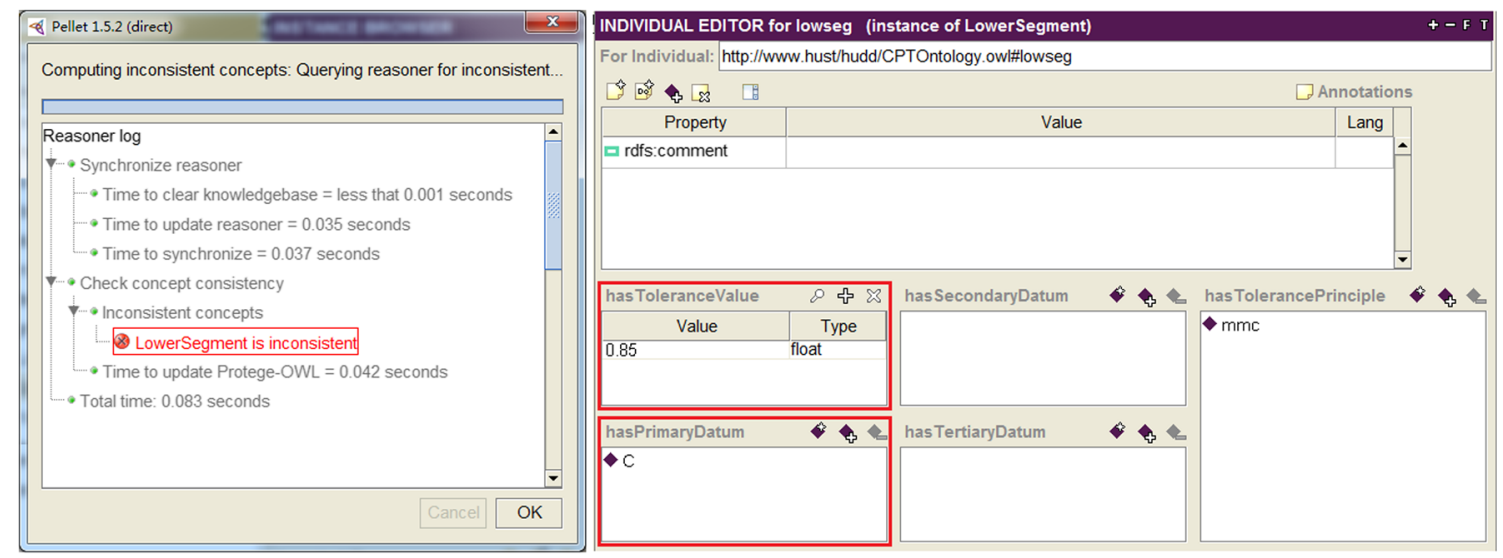

Fig. 6 Conformance checking result of the inconsistent drawing indication [50] 
lower segment. According to the definition of a composite positional tolerance, the tolerance value in the lower segment must be smaller than the tolerance value in the upper segment and the tertiary datum cannot be firstly repeated in the lower segment. Using description logic consistency decision algorithm, the two inconsistent places can be automatically checked out. The checking result is shown in Fig. 6. For more examples about the conformance checking, query, and reasoning on the ontology-based model, please refer to reference [48-50]. For the intrinsic conformance checking, query, and reasoning on other models, some additional techniques or tools are required. For instance, the conformance checking of the EXPRESS model can be implemented using an additional tool named STEP File Analyzer, which was developed by Lipman and Lubell [99] at National Institute of Standards and Technology.

- Does the representation model have self-learning and automatic update capabilities? All of the ten different kinds of representation models were manually established by domain experts. Thus, none of them has self-learning and automatic update capabilities.

Based on the above comparison, it is believed that an ideal representation model of tolerance information should have three levels of capabilities: primary capabilities, intermediate capabilities, and advanced capabilities, which are depicted in Fig. 7. The reasons are explained as follows:

- Primary capabilities. An ideal tolerance information representation model should firstly include a systematic representation of product geometry conformed to standards and unambiguously represent tolerance information syntax, because all types of tolerances are geometrically defined in existing tolerancing standards (e.g., ISO 1101 [100], ASME Y14.5 [101]) and these capabilities are the most fundamental requirements for a tolerance information representation model [59]. Further, an ideal model should be directly human-readable and computerreadable, since the purpose of tolerance information representation is to use computers to handle tolerance information, which requires the interactions of domain experts and computers.

- Intermediate capabilities. Tolerance information is represented as the symbol annotations of CAD models in most of the current commercial CAD systems. Even though using such representation form can establish a syntactically correct representation model of tolerance information, it cannot represent the semantics of tolerance information explicitly. For this reason, tolerance information is not really computer-interpretable in most of the current commercial CAD systems, which causes the incorrect use of tolerance information and impedes the implementation of automatic tolerancing and the integration of CAD and CAT. Conversely, if the semantics of tolerance information are explicitly represented in a CAD system, automatic checking of the conformance of tolerancing schemes, semantic query of tolerance information, automatic generation of tolerancing schemes, and exchange of tolerance information semantics would become reality in this CAD system. Further, semantics can offer a useful tool to improve the interchangeability of a representation model of tolerance information throughout different applications during the whole product lifecycle, which makes it
Fig. 7 Three levels of capabilities of an ideal tolerance information representation model

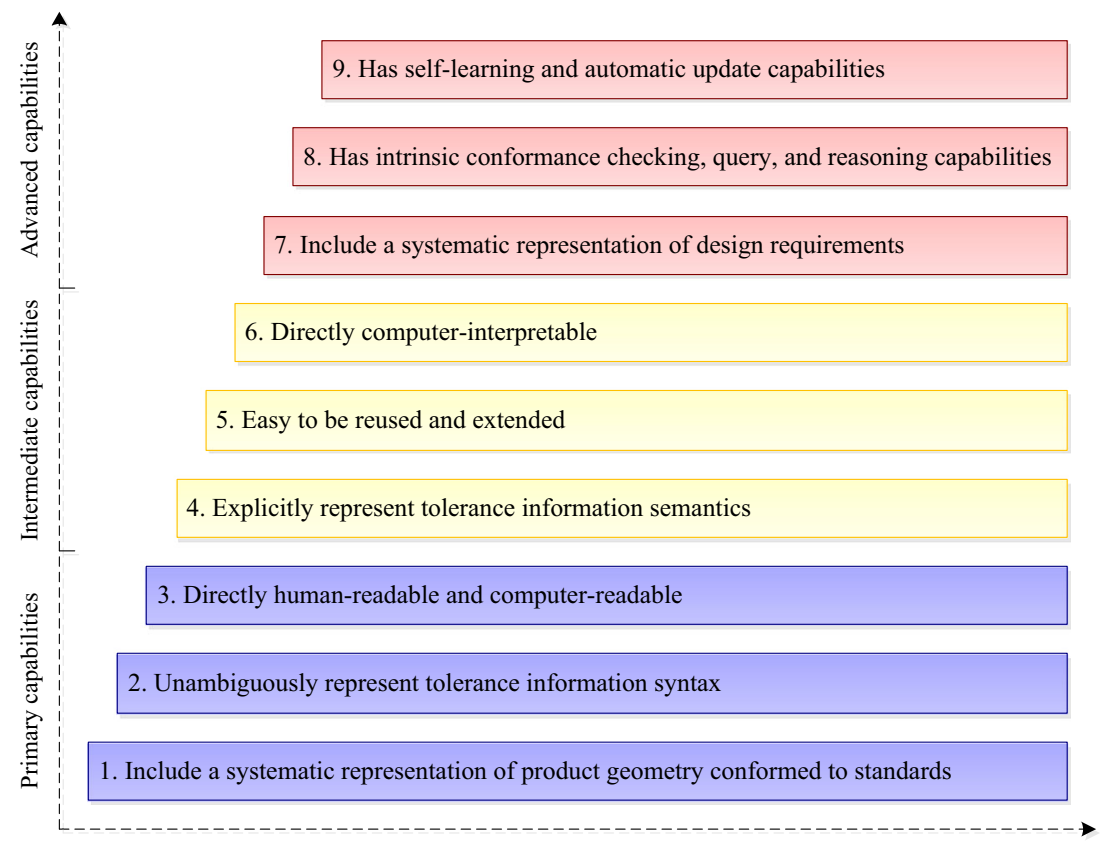


possible to directly use this representation model in manufacturing and measurement. In addition to semantics, an ideal representation model should be easy to be reused and extended and directly computer-interpretable, since an important purpose of establishing a representation model is to use it and good computer-interpretability will bring a high level of intelligence to CAT.

- Advanced capabilities. An ideal representation model should include a systematic representation of design requirements, because considering the representation of design requirements is very important for tolerance information representation and there is yet no evidence that an existing representation model has included a systematic representation of design requirements. Further, a representation model will be more convenient to use if it has intrinsic conformance checking, query, and reasoning capabilities. Finally, if a representation model has selflearning and automatic update capabilities, domain experts will not need to manually update it, which will bring great intelligence and convenience to CAT.

\section{Potential research directions}

Now, there is one last question Q5. Based on the analysis of the state-of-the-art in Sect. 2 and the comparison in Sect. 3, the following future research directions in tolerance information representation are suggested:

- Improvements of the EXPRESS model. For industry application, the EXPRESS model is still a relatively new representation model and would still be used in industry for a long time in the future [53]. Thus, a primary work is to study its improvements on the basis of its limitations, rather than to study how to completely replace it. According to the literature, a major limitation of the existing EXPRESS models is that they have great difficulty in explicitly representing tolerance information semantics, because the EXPRESS language is unable to represent information semantics [57] and they will not contain any representation of tolerance information semantics [58]. Aiming at this limitation, several researchers (e.g., Barbau et al. [102], Sarigecili [57]) presented to directly transform the existing EXPRESS models into OWL ontologies. They have successfully transformed the EXPRESS models AP 203 and AP 214 into OWL ontologies and extended and applied such ontologies to represent the semantics of tolerance specifications in tolerance analysis. However, the transformed OWL ontologies do not contain the representation of the detailed semantics of tolerance information, since both of the EXPRESS models AP 203 and AP 214 have not yet contained the representation of such semantics. It is still of necessity to study the improvements of the existing EXPRESS models with a representation capability of tolerance information semantics.

- Representation of tolerance information semantics. The tolerance information in current commercial CAD systems lacks explicit semantics. Although ontology-based model has capability to represent such semantics, most instantiations of the model are still manually carried out by domain experts, which is not only time consuming, error-prone, and tedious, but also requires a great deal of know-how [82]. It thus appears that the ontology-based model is not an ideal representation model. It is still of significance to study how to represent tolerance information semantics in a more effective way.

- Representation of the effects of design requirements, process, and material on tolerances. In tolerance information representation of a product, the design requirements, process, and material of the product require being synthetically considered, because all of these elements affect the tolerances of the product [88]. The existing ten kinds of representation models have involved systematic representations of the effect of product geometry on tolerances. They have not yet provided a systematic representation of the effects of design requirements, process, and material on tolerances. To allow a complete representation of a product, such representation needs to be added.

- Self-learning and automatic update of tolerance information representation models. All of the existing ten kinds of representation models were manually established by domain experts. If a user ought to reuse and extend one of these representation models, the user must firstly learn the principle of this representation model and then manually extend it for specific use. These tasks are not only time consuming, but also may lead to redundancies and human errors. If the representation model has self-learning and automatic update capabilities, the tasks will be automatically completed by computers and the redundancies and human errors will naturally be avoided.

- Standardization of tolerance information representation models. The standardization of a tolerance information representation model is the basis for realizing its industry application [53]. Among the existing ten kinds of representation models, only the EXPRESS model has been standardized. To implement the industry application of the remaining nine kinds of representation models, the study of the possibility of their standardization is an indispensable work.

- Integration of tolerance information representation models with commercial CAD systems. The integration of a representation model with commercial CAD systems can greatly facilitate the application of the representation model. Among the existing ten kinds of representation models, the EXPRESS model has been used in most 
current commercial CAD systems [80]; the TTRS model has been applied in CATIA [60]; and the category theory model has been integrated with AutoCAD and SolidWorks [43]. There is yet no evidence that the remaining seven kinds of representation models have been integrated with commercial CAD systems. To facilitate the application of these representation models, it is necessary to study such integration.

\section{Conclusion}

In this paper, a review of representation models of tolerance information has been presented. This review firstly conducted an in-depth analysis of existing ten different kinds of representation models. It then made a detailed comparison among them. Finally, the review pointed out some research perspectives in tolerance information representation.

Acknowledgements The authors would like to appreciate the insightful comments from the three anonymous reviewers for the improvement of the paper. The authors would like to acknowledge the financial supports by the National Natural Science Foundation of China (No.51475190), the Hubei Provincial Natural Science Foundation of China (No.2015CFA109), the Doctoral Dissertation Innovation Foundation of Huazhong University of Science and Technology, and the National Scholarship of China Scholarship Council.

Open Access This article is distributed under the terms of the Creative Commons Attribution 4.0 International License (http:// creativecommons.org/licenses/by/4.0/), which permits unrestricted use, distribution, and reproduction in any medium, provided you give appropriate credit to the original author(s) and the source, provide a link to the Creative Commons license, and indicate if changes were made.

\section{References}

1. Bjørke Ø (1989) Computer-aided tolerancing, 2nd edn. ASME Press, New York

2. Requicha AAG, Chan SC (1986) Representation of geometric features,tolerances, and attributes in solid modelers based on constructivegeometry. IEEE J Robot Autom 2(3):156-166. https://doi.org/10.1109/JRA.1986.1087053

3. Jayaraman R, Srinivasan V (1989) Geometric tolerancing: I. Virtual boundary requirements. IBM J Res Dev 33(2):90-104. https://doi.org/10.1147/rd.332.0090

4. Etesami F (1993) A mathematical model for geometric tolerances. ASME Trans J Mech Des 115(1):81-86. https://doi.org/10.1115/ 1.2919329

5. Hillyard RC, Braid IC (1978) Analysis of dimensions and tolerances in computer-aided mechanical design. Comput Aided Des 10(3):161-166. https://doi.org/10.1016/0010-4485(78)90140-9

6. Turner JU, Wozny MJ (1987) Tolerances in computer-aided geometric design. Vis Comput 3(4):214-226. https://doi.org/10.1007/ BF01952828

7. Gossard DC, Zuffante RP, Sakurai H (1988) Representing dimensions, tolerances, and features in MCAE systems. IEEE Comput Graph Appl 8(2):51-59. https://doi.org/10.1109/38.503
8. Hoffmann P (1982) Analysis of tolerances and process inaccuracies in discrete part manufacturing. Comput Aided Des 14(2):8388. https://doi.org/10.1016/0010-4485(82)90172-5

9. Turner JU, Wozny MJ (1990) The M-space theory of tolerances. Proc 16th ASME Des Autom Conf. American Society of Mechanical Engineers, New York, p 187-198

10. Martinsen K (1993) Vectorial tolerancing for all types of surfaces. Proc 19th ASME Des Autom Conf. American Society of Mechanical Engineers, New York, p 187-198

11. Chase KW, Gao J, Magleby SP, Sorenson CD (1996) Includinggeometric feature variations in tolerance analysis of mechanicalassemblies. IIE Trans 28(10):795-808. https://doi. org/10.1080/15458830.1996.11770732

12. Desrochers A, Riviere A (1997) A matrix approach to the representation of tolerance zones and clearances. Int J Adv Manuf Technol 13(9):630-636. https://doi.org/10.1007/BF01350821

13. Sacks E, Joskowicz L (1998) Parametric kinematic tolerance analysis of general planar systems. Comput Aided Des 30(9):707-714. https://doi.org/10.1016/S0010-4485(98)00024-4

14. Wang Y (2008) Semantic tolerance modeling with generalized intervals. ASME trans J Mech Des 130(8):081701. https://doi. org/10.1115/1.2936900

15. Shah JJ, Yan Y, Zhang BC (1998) Dimension and tolerance modeling and transformations in feature based design and manufacturing. J Intell Manuf 9(5):475-488. https://doi.org/10. 1023/A:1008856818686

16. Roy U, Li B (1998) Representation and interpretation of geometric tolerances for polyhedral objects I: form tolerances. Comput Aided Des 30(2):151-161. https://doi.org/10.1016/S00104485(97)00088-2

17. Roy U, Li B (1999) Representation and interpretation of geometric tolerances for polyhedral objects II: size, orientation and position tolerances. Comput Aided Des 31(4):273-285. https://doi.org/10. 1016/S0010-4485(99)00028-7

18. Davidson JK, Mujezinovic A, Shah JJ (2002) A new mathematicalmodel for geometric tolerances as applied to round faces. ASME Trans J Mech Des 124(4):609-622. https://doi.org/ $10.1115 / 1.1497362$

19. Mujezinovic A, Davidson JK, Shah JJ (2004) A new mathematicalmodel for geometric tolerances as applied to polygonal faces. ASME Trans J Mech Des 126(3):504-518. https://doi. org $/ 10.1115 / 1.1701881$

20. Ameta G, Davidson JK, Shah JJ (2007) Tolerance-maps applied toa point-line cluster of features. ASME Trans J Mech Des 129(8): 782-792. https://doi.org/10.1115/1.2717226

21. Liu Y, Yang J, Wu Z, Gao S (2001) Survey of modeling and representation of tolerance information in CAD system. $\mathrm{J}$ Comput-Aided Des Comput Graph 13(11):1048-1055

22. Hong YS, Chang TC (2002) A comprehensive review of tolerancing research. Int J Prod Res 40(11):2425-2459. https:// doi.org/10.1080/00207540210128242

23. Marziale M, Polini W (2009) A review of two models for tolerance analysis of an assembly: vector loop and matrix. Int J Adv Manuf Technol 43(11):1106-1123. https://doi.org/10.1007/ s00170-008-1790-0

24. Marziale M, Polini W (2011) Review of variational models for tolerance analysis of an assembly. Proc Inst Mech Eng B J Eng Manuf 225(3):305-318. https://doi.org/10.1177/2041297510394107

25. Marziale M, Polini W (2011) A review of two models for tolerance analysis of an assembly: Jacobian and torsor. Int J Comput Integr Manuf 24(1):74-86. https://doi.org/10.1080/0951192X. 2010.531286

26. Ameta G, Serge S, Giordano M (2011) Comparison of spatial math models for tolerance analysis: tolerance-maps, deviation domain, and TTRS. ASME Trans J Comput Inf Sci Eng 11(2): 021004. https://doi.org/10.1115/1.3593413 
27. Polini W (2012) Taxonomy of models for tolerance analysis in assembling. Int J Prod Res 50(7):2014-2029. https://doi.org/10. 1080/00207543.2011.576275

28. Chen H, Jin S, Li Z, Lai X (2014) A comprehensive study of three dimensional tolerance analysis methods. Comput Aided Des 53(8):1-13. https://doi.org/10.1016/j.cad.2014.02.014

29. ISO10303-203 (2011) Industrial automation systems and integration - product data representation and exchange - part 203: application protocol: configuration controlled $3 \mathrm{D}$ design of mechanical parts and assemblies. International Organization for Standardization, Geneva

30. ISO10303-214 (2010) Industrial automation systems and integration-product data representation and exchange-part 214: Application protocol: Core data for automotive mechanical design processes. International Organization for Standardization, Geneva

31. ISO10303-242 (2014) Industrial automation systems and integration - product data representation and exchange - part 242: application protocol: managed model-based 3D engineering. International Organization for Standardization, Geneva

32. Requicha AAG, Voelcker HB (1982) Solid modeling: a historical summary and contemporary assessment. IEEE Comput Graph Appl 2(2):9-24. https://doi.org/10.1109/MCG.1982.1674149

33. Juster NP (1992) Modeling and representation of dimensions and tolerances: a survey. Comput Aided Des 24(1):3-17. https://doi. org/10.1016/0010-4485(92)90086-P

34. Roy U, Liu CR (1988) Feature-based representational scheme of a solid modeler for providing dimensioning and tolerancing information. Robot Comput-Integr Manuf 4(3):333-345

35. Guilford J, Turner J (1993) Representational primitives for geometric tolerancing. Comput Aided Des 25(9):577-586. https://doi. org/10.1016/0010-4485(93)90073-W

36. Wilhelm RG, Lu SCY (1992) Computer methods for tolerance design. World Scientific, River Edge. https://doi.org/10.1142/1735

37. Desrochers A, Clement A (1994) A dimensioning and tolerancing assistance model for CAD/CAM systems. Int J Adv Manuf Technol 9(6):352-361. https://doi.org/10.1007/BF01748479

38. Rachuri S, Han YH, Feng SC, Roy U, Wang F, Sriram RD, Lyons KW (2004) Object-oriented representation of electro-mechanical assemblies using UML. National Institute of Standards and Technology, Gaithersburg

39. Zhao X, Pasupathy TK, Wilhelm RG (2006) Modeling and representation of geometric tolerances information in integrated measurement processes. Comput Ind 57(4):319-330. https://doi.org/ 10.1016/j.compind.2005.09.004

40. Lu W, Jiang X, Liu X, Qi Q, Scott PJ (2010) Modeling the integration between specifications and verification for cylindricity based on category theory. Meas Sci Technol 21(11):115107. https://doi.org/10.1088/0957-0233/21/11/115107

41. Xu Y, Xu Z, Jiang X, Scott P (2011) Developing a knowledgebased system for complex geometrical product specification (GPS) data manipulation. Knowl-Based Syst 24(1):10-22. https://doi.org/10.1016/j.knosys.2010.05.002

42. Qi Q, Jiang X, Scott PJ (2012) Knowledge modeling for specifications and verification in areal surface texture. Precis Eng 36(2): 322-333. https://doi.org/10.1016/j.precisioneng.2011.12.002

43. Qi Q, Scott PJ, Jiang X, Lu W (2014) Design and implementation of an integrated surface texture information system for design, manufacture and measurement. Comput Aided Des 57(12):4153. https://doi.org/10.1016/j.cad.2014.06.013

44. Dantan JY, Ballu A, Mathieu L (2008) Geometrical product specifications - model for product life cycle. Comput Aided Des 40(4):493-501. https://doi.org/10.1016/j.cad.2008.01.004

45. Zhang Y, Li Z, Xu L, Wang J (2011) A new method for automatic synthesis of tolerances for complex assemblies based on polychromatic sets. Enterp Inf Syst 5(3):337-358. https://doi.org/10.1080/ 17517575.2011.593004
46. Qin Y, Zhong Y, Huang M, Liu F (2014) An assembly tolerance representation model based on spatial relations for generating assembly tolerance types. Proc Inst Mech Eng C J Mech Eng Sci 228(6):1005-1020. https://doi.org/10.1177/0954406213495501

47. Fiorentini X, Gambino I, Liang VC, Foufou S, Rachuri R, Mani M, Bock C (2007) An ontology for assembly representation. National Institute of Standards and Technology, Gaithersburg

48. Lu W, Qin Y, Liu X, Huang M, Zhou L, Jiang X (2015) Enriching the semantics of variational geometric constraint data with ontology. Comput Aided Des 63(6):72-85. https://doi.org/10.1016/j. cad.2014.12.008

49. Qin Y, Lu W, Liu X, Huang M, Zhou L, Jiang X (2015) Description logic-based automatic generation of geometric tolerance zones. Int J Adv Manuf Tech 79(5):1221-1237. https://doi. org/10.1007/s00170-015-6839-2

50. Qin Y, Lu W, Qi Q, Li T, Huang M, Scott PJ, Jiang X (2017) Explicitly representing the semantics of composite positional tolerance for patterns of holes. Int J Adv Manuf Tech 90(5):21212137. https://doi.org/10.1007/s00170-016-9457-8

51. Roy U, Liu CR, Woo TC (1991) Review of dimensioning and tolerancing: representation and processing. Comput Aided Des 23(7):466-483. https://doi.org/10.1016/0010-4485(91)90045-X

52. Yu KM, Tan ST, Yuen MF (1994) A review of automatic dimensioning and tolerancing schemes. Eng Comput 10(2):63-80

53. Srinivasan V (2008) Standardizing the specification, verification, and exchange of product geometry: research, status and trends. Comput Aided Des 40(7):738-749. https://doi.org/10.1016/j.cad.2007.06.006

54. ISO10303-1 (1994) Industrial automation systems and integration - product data representation and exchange-part 1: Overview and fundamental principles. International Organization for Standardization, Geneva

55. ISO10303-11 (2004) Industrial automation systems and integration-product data representation and exchange - part 11: Description methods: The EXPRESS language reference manual. International Organization for Standardization, Geneva

56. ISO10303-21 (2002) Industrial automation systems and integration-product data representation and exchange-part 21: Implementation methods: Clear text encoding of the exchange structure. International Organization for Standardization, Geneva

57. Sarigecili MI, Roy U, Rachuri S (2014) Interpreting the semantics of GD\&T specifications of a product for tolerance analysis. Comput Aided Des 47(2):72-84. https://doi.org/10.1016/j.cad. 2013.09.002

58. Feeney AB, Frechette SP, Srinivasan V (2015) A portrait of an ISO STEP tolerancing standard as an enabler of smart manufacturing systems. ASME Trans J Comput Inf Sci Eng 15(2):021001

59. Liu Y, Gao S, Wu Z, Yang J (2003) Hierarchical representation model and its realization of tolerance based on feature. Chin $\mathrm{J}$ Mech Eng 39(3): 1-7

60. Prisco U, Giorleo G (2002) Overview of current CAT systems. Integr Comput-Aided Eng 9(4):373-387

61. Clement A, Riviere A, Serre P, Valade C (1998) The TTRSs: 13 constraints for dimensioning and tolerancing. Proc 5th CIRP Int Sem Comput-aided Tolerancing. Chapman and Hall, London, p 122-131

62. Desrochers A, Maranzana R (1995) Constrained dimensioning and tolerancing assistance for mechanisms. Proc 4th CIRP Int Sem Comput-Aided Tolerancing. Springer Netherlands, Houten, p 17-30

63. Salomons OW, Poerink HJ, Haalboom FJ, Van Slooten F, Van Houten FJ, Kals HJ (1996) A computer aided tolerancing tool I: tolerance specification. Comput Ind 31(2):161-174. https://doi. org/10.1016/0166-3615(96)00046-2

64. Toulorge H, Riviere A, Bellacicco A, Sellakh R (2003) Towards a digital functional assistance process for tolerancing. ASME Trans J Comput Inf Sci Eng 3(1):39-44. https://doi.org/10.1115/1.1570452 
65. Jaballi K, Bellacicco A, Louati J, Riviere A, Haddar M (2011) Rational method for 3D manufacturing tolerancing synthesis based on the TTRS approach "R3DMTSyn". Comput Ind 62(5): 541-554. https://doi.org/10.1016/j.compind.2011.02.003

66. Zhang H, Cao Y, Wei Y, Yang J (2013) A concurrent design method for functional tolerance and structure based on the principle of decomposition and reconstitution. Proc 12th CIRP Int Sem Comput-aided Tolerancing. Procedia CIRP 10:194-202, DOI: https://doi.org/10.1016/j.procir.2013.08.031

67. Fenves SJ (2002) A Core product model for representing design information. National Institute of Standards and Technology, Gaithersburg

68. ASME Y14.5M (1994) Dimensioning and tolerancing. American Society of Mechanical Engineers, New York

69. Liu S, Mei J, Yue A, Lin Z (2004) XSDL: making xml semantics explicit. International Workshop on Semantic Web and Databases. Springer-Verlag Berlin Heidelberg, Heidelberg, pp 64-83

70. Wang Y, Scott PJ, Jiang X (2005) The structure of surface texture knowledge. J Phys: Conf Ser 13(1):1-4

71. Ballu A, Mathieu L, Dantan JY (2015) Formal language for GeoSpelling. ASME Trans J Comput Inf Sci Eng 15(2):021002

72. Hu J, Xiong G, Wu Z (2004) A variational geometric constraints network for a tolerance type specification. Int Adv Manuf Technol 24(3):214-222

73. Zhang Y, Li Z, Gao J, Hong J (2011) New reasoning algorithm for assembly tolerance specifications and corresponding tolerance zone types. Comput Aided Des 43(12):1606-1628. https://doi. org/10.1016/j.cad.2011.06.008

74. McGuinness DL, van Harmelen F (2004) OWL Web Ontology Language Overview. http://www.w3.org/TR/owl-features/

75. Horrocks I, Patel-Schneider PF, Boley H, Tabet S, Grosof B, Dean M (2004) SWRL: A Semantic Web Rule Language Combining OWL and RuleML. http://www.w3.org/Submission/SWRL/

76. Zhong Y, Qin Y, Huang M, Lu W, Chang L (2014) Constructing a meta-model for assembly tolerance types with a description logic based approach. Comput Aided Des 48(3):1-16. https://doi.org/ 10.1016/j.cad.2013.10.009

77. Zhong Y, Qin Y, Huang M, Lu W, Gao W, Du Y (2013) Automatically generating assembly tolerance types with an ontology-based approach. Comput Aided Des 45(11):12531275. https://doi.org/10.1016/j.cad.2013.06.006

78. Ahmed F, Han S (2015) Interoperability of product and manufacturing information using ontology. Concurr Eng Res Appl 23(3):265-278

79. Qin Y, Lu W, Qi Q, Liu X, Huang M, Scott PJ, Jiang X (2017) Towards an ontology-supported case-based reasoning approach for computer-aided tolerance specification. Knowl Based Syst. https://doi.org/10.1016/j.knosys.2017.11.013

80. Qin Y, Lu W, Qi Q, Liu X, Zhong Y, Scott PJ, Jiang X (2017) Status, comparison, and issues of computer-aided design model data exchange methods based on standardized neutral files and web ontology language file. ASME Trans J Comput Inf Sci Eng 17(1):010801

81. Chandrasegaran SK, Ramani K, Sriram RD, Horváth I, Bernard A, Harik RF, Gao W (2013) The evolution, challenges, and future of knowledge representation in product design systems. Comput Aided Des 45(2):204-228. https://doi.org/10.1016/j.cad.2012.08.006

82. Fortineau V, Paviot T, Lamouri S (2013) Improving the interoperability of industrial information systems with description logicbased models - the state of the art. Comput Ind 64(4):363-375. https://doi.org/10.1016/j.compind.2013.01.001

83. El Kadiri S, Kiritsis D (2015) Ontologies in the context of product lifecycle management: state of the art literature review. Int J Prod Res 53(18):5657-5668. https://doi.org/10.1080/00207543.2015.1052155
84. Ramos L (2015) Semantic web for manufacturing, trends and open issues: toward a state of the art. Comput Ind Eng 90(12): 444-460. https://doi.org/10.1016/j.cie.2015.10.013

85. Negri E, Fumagalli L, Garetti M, Tanca L (2016) Requirements and languages for the semantic representation of manufacturing systems. Comput Ind 81(9):55-66. https://doi.org/10.1016/j. compind.2015.10.009

86. Baader F, Calvanese D, McGuinness DL, Nardi D, PatelSchneider PF (2010) The description logic handbook: theory, implementation and applications, 2nd edn. Cambridge University Press, Cambridge

87. Horrocks I, Patel-Schneider PF, Van Harmelen F (2003) From SHIQ and RDF to OWL: the making of a web ontology language. J Web Semant 1(1):7-26. https://doi.org/10.1016/j.websem.2003.07.001

88. Armillotta A, Semeraro Q (2011) Geometric tolerance specification. Colosimo BM, Senin N, editors. Geometric tolerances: impact on product design, quality inspection and statistical process monitoring. Springer London, London, pp 3-37

89. Ballu A, Mathieu L (1999) Choice of functional specifications using graphs within the framework of education. Proc 6th CIRP Int Sem Comput-Aided Tolerancing. Springer Netherlands, Houten,p 197-206

90. Wang H, Roy U, Sudarsan R, Sriram RD, Lyons KW (2003) Functional tolerancing of a gearbox. Proc north am Manuf res Conf. National Institute of Standards and Technology, Gaithersburg

91. Anselmetti B (2006) Generation of functional tolerancing based on positioning features. Comput Aided Des 38(8):902-919. https://doi.org/10.1016/j.cad.2006.05.005

92. Armillotta A (2013) A method for computer-aided specification of geometric tolerances. Comput Aided Des 45(12):1604-1616. https://doi.org/10.1016/j.cad.2013.08.007

93. Cao Y, Zhang H, Li B, Wu Z, Yang J (2013) Study on functional specification scheme on interface based on positioning features. Proc Inst Mech Eng B J Eng Manuf 227(5):745-753. https://doi. org/10.1177/0954405413479112

94. CAx Implementor Forum (2017) CAx IF Implementation Forum. https://cax-if.org/vendor_info.php

95. ISO/IEC19501 (2005) Information technology—open distributed processing - unified modeling language (UML). International Organization for Standardization, Geneva

96. Bray T, Paoli J, Sperberg-McQueen CM, Mailer Y, Yergeau F (2008) Extensible Markup Language (XML) 1.0 (Fifth Edition). http://www.w3.org/TR/REC-xml/

97. Venkiteswaran A, Hejazi SM, Biswas D, Shah JJ, Davidson JK (2016) Semantic Interoperability of GD\&T Data Through ISO 10303 Step AP242. Proc ASME 2016 Int Des Eng Tech Conf Comput Inf Eng Conf. American Society of Mechanical Engineers, New York, p V02BT03A018

98. Baysal MM, Roy U, Sudarsan R, Sriram RD, Lyons KW (2005) Product information exchange using open assembly model: issues related to representation of geometric information. Proc ASME 2005 Int Mech Eng Congr Expo. American Society of Mechanical Engineers, New York, p 601-612

99. Lipman R, Lubell J (2015) Conformance checking of PMI representation in CAD model STEP data exchange files. Comput Aided Des 66(9):14-23. https://doi.org/10.1016/j.cad.2015.04.002

100. ISO 1101 (2012) Geometrical product specifications (GPS) - geometrical tolerancing - tolerances of form, orientation, location and run-out. International Organization for Standardization, Geneva

101. ASME Y14.5 (2009) Dimensioning and tolerancing. American Society of Mechanical Engineers, New York

102. Barbau R, Krima S, Rachuri S, Narayanan A, Fiorentini X, Foufou S, Sriram RD (2012) OntoSTEP: enriching product model data using ontologies. Comput Aided Des 44(6):575-590. https://doi. org/10.1016/j.cad.2012.01.008 